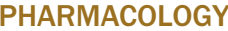

\section{Structure-based design for selective inhibition lowers toxicity}

A compound that inhibits the growth of BCL-2-dependent tumours has been re-engineered to prevent the concurrent inhibition of the BCL- $\mathrm{X}_{\mathrm{L}}$ protein that protects platelets, report Andrew Souers and colleagues in Nature Medicine.

Apoptosis is tightly controlled by pro-death and pro-survival proteins. Shifting the balance towards pro-survival proteins is one mechanism by which cancer cells are able to evade apoptosis. BCL-2 is a protein regulator of apoptosis that has a major role in the survival of a number of lymphoid malignancies. BCL- $\mathrm{X}_{\mathrm{L}}$ is a related pro-survival protein that is associated with a number of haematological malignancies and solid-tumours, and is also is the primary pro-survival factor in platelets.

\section{4 ...higher concentrations of} the drug can be administered without inducing toxicity 77

The investigators had previously described navitoclax - a molecule with a high affinity for both BCL-2 and BCL- $\mathrm{X}_{\mathrm{L}}$. Navitoclax has antitumour activity in lymphoid malignancies that are dependent on BCL-2 for survival, but also reduces the number of circulating platelets, which limits the safe administrative dose of navitoclax in patients. "We wanted to engineer a compound that inhibits the BCL-2 protein that protects cancer cells, but does not inhibit the BCL- $\mathrm{X}_{\mathrm{L}}$ protein that protects platelets" explains Souers, an Associate Director for AbbVie.

BCL-2 and BCL- $\mathrm{X}_{\mathrm{L}}$ both contain similar $\mathrm{BH} 3$-binding domains that complicate the design of BCL-2-selective inhibitors. The team used X-ray crystallography to visualize protein interactions and noted that "an early and less potent analogue crystallized with the BCL-2 protein in such a way that a new means of interacting with the protein was discovered" says Souers, "the observation gave us significant insight into how a new compound could be made such that the activity against BCL- $\mathrm{X}_{\mathrm{L}}$ would be substantially reduced."

By removing and replacing key binding elements, the team were able to manipulate the structure of the molecule. The resulting compound, ABT-199, induced apoptosis in various cancer cell lines and resulted in tumour regression in vivo while sparing platelets, meaning that higher concentrations of the drug can be administered without inducing toxicity. Notably, administration of a single dose of ABT-199 in three patients with chronic lymphoid leukaemia resulted in rapid tumour lysis revealing the potential of this agent, and this structure-based design approach, in cancer therapy.

\section{Bryony Jones}

Original article Souers, A. J. et al. ABT-199, a potent and selective BCL-2 inhibitor, achieves antitumor activity while sparing platelets Nat. Med. doi:10.1038/nm.3048 